$02,09,13$

\title{
Источник терагерцевого излучения в открытое пространство на основе распределенного джозефсоновского перехода
}

\author{
( Н.В. Кинев, К.И. Рудаков, Л.В. Филиппенко, В.П. Кошелец, А.М. Барышев \\ Институт радиотехники и электроники им. В.А. Котельникова РАН, \\ Москва, Россия \\ E-mail: nickolay@hitech.cplire.ru
}

Поступила в Редакцию 26 марта 2020 г.

В окончательной редакции 26 марта 2020 г.

Принята к публикации 2 апреля 2020 г.

\begin{abstract}
Предложен и экспериментально исследован источник электромагнитного излучения терагерцевого (ТГц) диапазона частот на основе туннельного распределенного джозефсоновского перехода. Выходное излучение передается в открытое пространство посредством щелевой антенны, размещенной на одной микросхеме с переходом и согласованной с собирающей линзой. Изготовлены и исследованы несколько конструкций генератора, рассчитанных на работу в частотных диапазонах $250-410 \mathrm{GHz}, 330-530 \mathrm{GHz}$ и $390-700 \mathrm{GHz}$ с возможностью непрерывной перестройки частоты. Исследование амплитудно-частотных характеристик выходного излучения со спектральным разрешением порядка $0.1 \mathrm{MHz}$ проведено при помощи ТГц-спектрометра на основе сверхпроводникового приемника. Исследование характеристик передающей антенны во всей полосе пропускания проведено при помощи охлаждаемого высокочувствительного болометра на основе кремния. Экспериментальные результаты соответствуют численным расчетам.
\end{abstract}

Ключевые слова: источники излучения терагерцевого диапазона, эффект Джозефсона, щелевая антенна, гармонический смеситель.

DOI: $10.21883 /$ FTT.2020.09.49757.02H

\section{1. Введение}

Источники терагерцевого (ТГц) излучения востребованы на сегодняшний день в ряде научных задач, таких как гетеродинное детектирование сверхслабых сигналов для радиоастрономических исследований, спектроскопия высокого разрешения в ТГц-диапазоне. Спектроскопия газов и живых тканей широко используется в биологии и медицине, а также в экологическом мониторинге и системах безопасности. Распределенный джозефсоновский переход (РДП) работает как источник электромагнитного излучения ТГц-диапазона $[1]$, частота которого $f$ однозначно определена постоянным напряжением смещения на переходе $V_{d c}$ в соответствии с соотношением Джозефсона

$$
h f=2 e V_{d c},
$$

где $h$ - постоянная Планка, $e-$ заряд электрона. Путем приложения внешнего локального магнитного поля и задания тока смещения через РДП, внутри туннельного перехода создается однонаправленный поток магнитных вихрей (квантов магнитного потока) флаксонов. За последние десятилетия конструкция такого перехода была существенно доработана с целью его применения именно в качестве широко перестраиваемого генератора ТГц-диапазона [2], а его спектральные характеристики были тщательно изучены [3] с использованием гармонического смесителя для понижения частоты выходного излучения до диапазона $0.1-1 \mathrm{GHz}$ и стабилизации излучения на основе системы фазовой автоподстройки частоты (ФАПЧ). Сигнал в диапазоне частот до $1 \mathrm{GHz}$ может быть измерен непосредственно при помощи лабораторного анализатора спектра с разрешением порядка $1 \mathrm{~Hz}$. На сегодняшний день достигнуты следующие выходные характеристики источника на основе РДП: частота генерации от 300 до $700 \mathrm{GHz}$ с возможностью непрерывной перестройки, ширина линии генерации без использования системы ФАПЧ находится в пределах между 0.5 и $15 \mathrm{MHz}$ в зависимости от режима работы генератора, реализована фазовая синхронизация выходного излучения в узком центральном пике шириной порядка $40 \mathrm{kHz}$ со спектральным качеством до 97\%, уровень выходной мощности достаточен для задач гетеродинного приема. Набор таких характеристик позволил успешно реализовать РДП в качестве генератора-гетеродина в составе сверхпроводникового приемника для полетной миссии Terahertz Limb Sounder (TELIS) [4-5]. Указанный приемник основан на смесителе в виде туннельного перехода „сверхпроводник-изолятор-сверхпроводник“ (СИС) и назван интегральным приемником благодаря интеграции РДП на одной микросхеме с СИС-смесителем.

Некоторое время назад нами была предложена и успешно реализована концепция интеграции РДП на одной микросхеме с передающей щелевой антенной [6-12] с целью передачи ТГц-излучения в открытое пространство. Частотные характеристики различных конструкций передающей антенны, рассчитанных на разные частотные диапазоны (импеданс, доля излученной мощности, диаграмма направленности), исследованы и представлены в работах [6,7]. Затем, с целью фазовой синхронизации излучения, мы дополнительно встроили в инте- 
гральную микросхему гармонический смеситель (ГС), работающий в цепи обратной связи с РДП совместно с системой ФАПЧ. Согласование по мощности ГС и РДП были подробно исследованы в работах [8-11], а спектральные характеристики излучения в открытое пространство исследованы в работах $[9,12]$. В данной работе мы провели более глубокие исследования выходного излучения в открытое пространство посредством двух различных экспериментальных установок. В разделе 2 описан технологический процесс изготовления экспериментальных образцов и приведены характеристики полученных туннельных СИС-структур по постоянному току. Исследование амплитудно-частотных характеристик (АЧХ) со спектральным разрешением порядка $0.1 \mathrm{MHz}$ проведено при помощи ТГц-спектрометра на основе сверхпроводникового интегрального приемника; результаты представлены в разделе 3. Частотная зависимость интегральной мощности излучения в открытое пространство во всем диапазоне генерации РДП, фактически определяемая характеристиками передающей антенны, исследована при помощи охлаждаемого высокочувствительного болометра на основе кремния; результаты приведены в разделе 4.

\section{2. Изготовление экспериментальных образцов ТГц-источника}

Была изготовлена серия экспериментальных микросхем размером $4 \times 4 \mathrm{~mm}^{2}$ на кремниевой подложке толщиной около $0.5 \mathrm{~mm}$. Сверхпроводниковые туннельные структуры выполнены на основе $\mathrm{Nb} / \mathrm{AlO}_{x} / \mathrm{Nb}$, технологический процесс формирования таких структур более подробно описан в работах [13-14]. Для предотвращения подтрава подложки в процессе плазменного травления при формировании туннельных переходов используется осаждение буферного слоя $\mathrm{Al}_{2} \mathrm{O}_{3}$ толщиной около $100 \mathrm{~nm}$. На следующем этапе производится осаждение трехслойной структуры $\mathrm{Nb} / \mathrm{AlO}_{x} / \mathrm{Nb}$ методами магнетронного напыления. Геометрия электродов формируется посредством „взрывной“ литографии. Толщина нижнего и верхнего электродов на основе ниобия составляет около $200 \mathrm{~nm}$ и $400-450 \mathrm{~nm}$ соответственно. Микрополосковые линии передачи выполнены на основе $\mathrm{Nb} / \mathrm{SiO}_{2} / \mathrm{Nb}$ с толщиной изолятора порядка $400 \mathrm{~nm}$. Вскрытие контактов к структурам производится также посредством „взрывной“ литографии, после чего напыляются золотые контактные площадки. В результате были изготовлены экспериментальные образцы трех интегральных конструкций, включающих РДП, щелевую антенну и ГС, рассчитанных на перестройку рабочей частоты в диапазонах $250-410 \mathrm{GHz}$ (далее - конструкция К-300), 330-530 GHz (конструкция К-450) и $390-700 \mathrm{GHz}$ (конструкция К-550). Числом в названии конструкций для ясности обозначена центральная частота рабочего диапазона, выраженная в гигагерцах. Размеры РДП в составе экспериментальных микросхем составляют $16-400 \mu \mathrm{m}^{2}$ для конструкций К-450

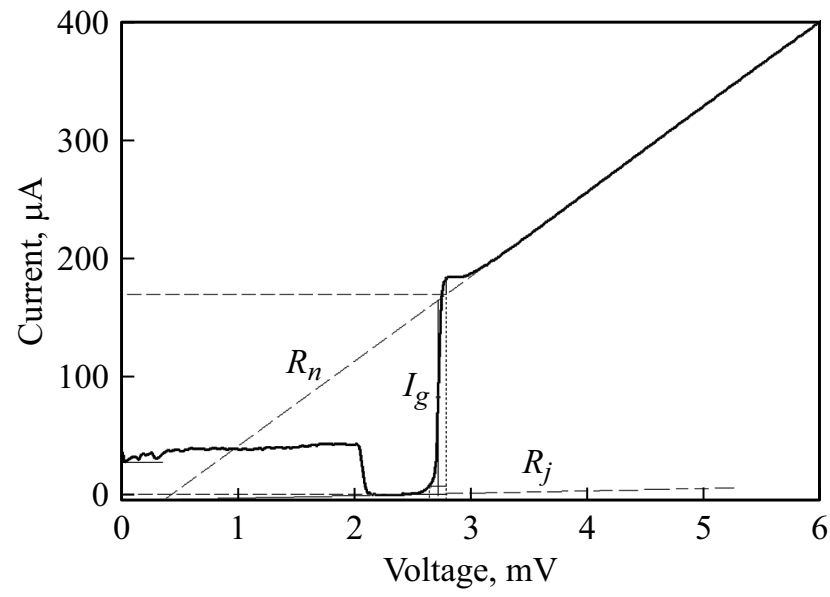

Рис. 1. ВАХ туннельного перехода на основе $\mathrm{Nb} / \mathrm{AlO}_{x} / \mathrm{Nb}$ площадью $1.4 \mu \mathrm{m}^{2}$. Параметры перехода: нормальное сопротивление $R_{n}=14.1 \Omega$; отношение „подщелевого“] сопротивления $R_{j}$, характеризующего ток утечки, к нормальному сопротивлению $R_{j} / R_{n}=34.7$; щелевое напряжение $V_{g}=2.71 \mathrm{mV}$; скачок тока при щелевом напряжении $I_{g}=161 \mu \mathrm{A}$. Вспомогательные пунктирные линии приведены для наглядного обозначения параметров.

и К-550, и $16 \times 700 \mu \mathrm{m}^{2}$ для конструкции К-300. Тестирование экспериментальных образцов по постоянному току продемонстрировало высокое качество туннельных структур. На рис. 1 представлена вольт-амперная характеристика (BAX) перехода площадью $1.4 \mu \mathrm{m}^{2}$ на основе $\mathrm{Nb} / \mathrm{AlO}_{x} / \mathrm{Nb}$ в составе конструкции К-300 при температуре $4.2 \mathrm{~K}$. Участок BAX при напряжениях ниже $2 \mathrm{mV}$ характеризуется критическим током СИС-перехода, который в данном случае не подавлен. Плотность туннельного тока СИС-структуры составляет около $6.5 \mathrm{kA} / \mathrm{cm}^{2}$.

\section{3. Исследование спектральных характеристик}

АЧХ выходного излучения были исследованы со спектральным разрешением лучше $0.1 \mathrm{MHz}$ при помощи спектрометра на основе сверхпроводникового интегрального приемника (СИП) [4,5]. Схема экспериментальной установки показана на рис. 2. Источник и приемник установлены в два независимых заливных гелиевых криостата: верхняя часть схемы представляет собой криостат № 1 с исследуемым генератором, нижняя часть схемы - криостат № 2 с микросхемой приемника (на схеме, SIR - сокр. от „superconducting integrated receiver" ${ }^{6}$. Принцип работы СИП детально описан, например, в работах [15-16]. В установке использовано одновременно два РДП (на схеме, FFO - сокр. от „flux flow oscillator"): излучение первого (FFO \#1) является объектом исследования в данной работе, второй (FFO \#2) является гетеродином СИП. Форма спектральной линии излучения регистрируется 


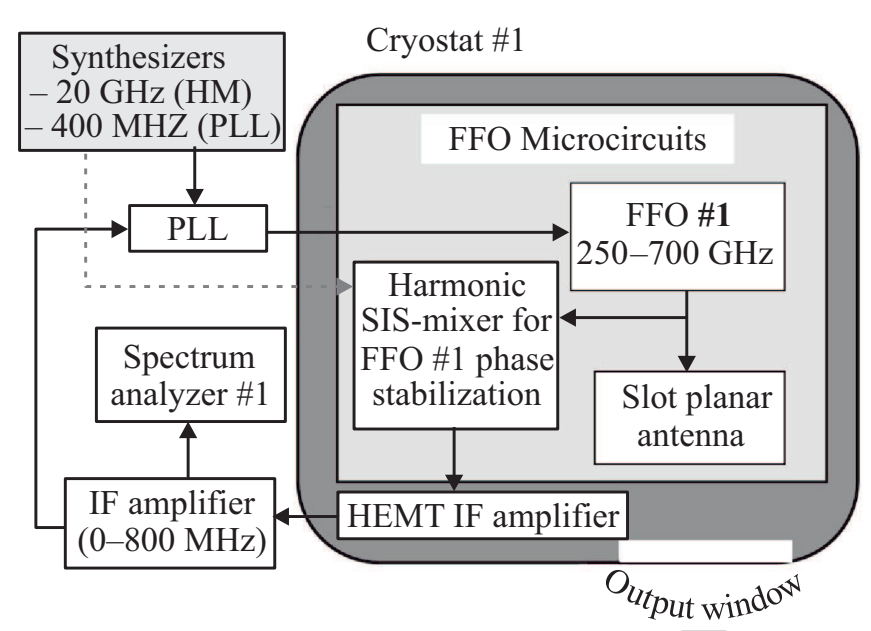

$\mathrm{THz}$ radiation

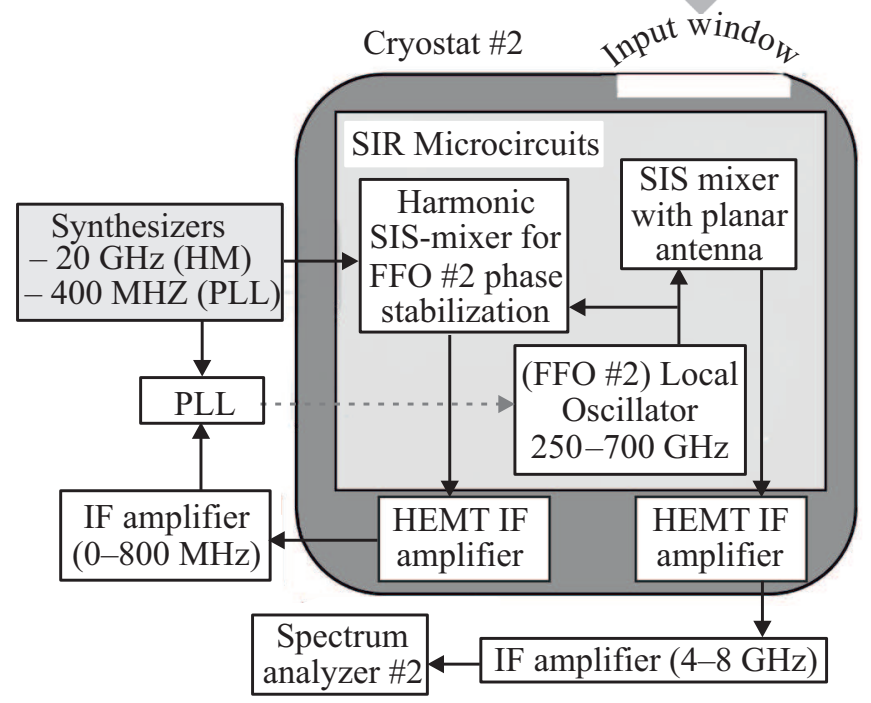

Рис. 2. Схема установки по исследованию спектральных характеристик источника на основе РДП, расположенного в криостате № 1, в открытое пространство при помощи терагерцового спектрометра на основе СИП, расположенного в криостате № 2 .

двумя независимыми способами: анализатором спектра № 1 через цепь обратной связи для стабилизации излучения при помощи ГС и системы ФАПЧ (на схеме, PLL - сокр. от „phase locking loop“) на промежуточной частоте (ПЧ) гармонического смесителя в диапазоне 0-800 MHz, и анализатором спектра № 2 на выходе СИП в диапазоне ПЧ 4-8 GHz. В трактах ПЧ как ГС, так и СИС-смесителя использованы полупроводниковые сверхвысокочастотные (СВЧ) усилители (на схеме, IF amplifier - сокр. от, intermediate frequency amplifier ${ }^{66}$ ) с усилением порядка $30 \mathrm{~dB}$ для охлаждаемых и порядка $60 \mathrm{~dB}$ для неохлаждаемых усилителей, находящихся при комнатной температуре, так что суммарное усиление выходного СВЧ-сигнала составляет порядка $90 \mathrm{~dB}$. Следует отметить, что при помощи такой установки возможно исследование излучения в открытое пространство только в рабочем диапазоне частот СИП 480-730 GHz, поэтому описанное исследование проведено для конструкций К-450 и К-550. Исследование спектральных характеристик для конструкции К-300 по-прежнему возможно при помощи анализатора спектра № 1, без использования внешнего приемника.

На рис. 3 представлены результаты измерения АЧХ излучения экспериментального образца конструкции К-300 при помощи анализатора спектра № 1 на четырех частотах с шагом $25 \mathrm{GHz}: 250,275,300$ и $325 \mathrm{GHz}$. Спектры излучения на рис. 3, $a$ зарегистрированы в режиме автономной генерации, на рис. $3, b-$ в режиме фазовой стабилизации излучения к опорному генератору частотой $400 \mathrm{MHz}$. Ширина представленных линий излучения в автономном режиме составила от 0.4 до $2.9 \mathrm{MHz}$, а спектральное качество в режиме
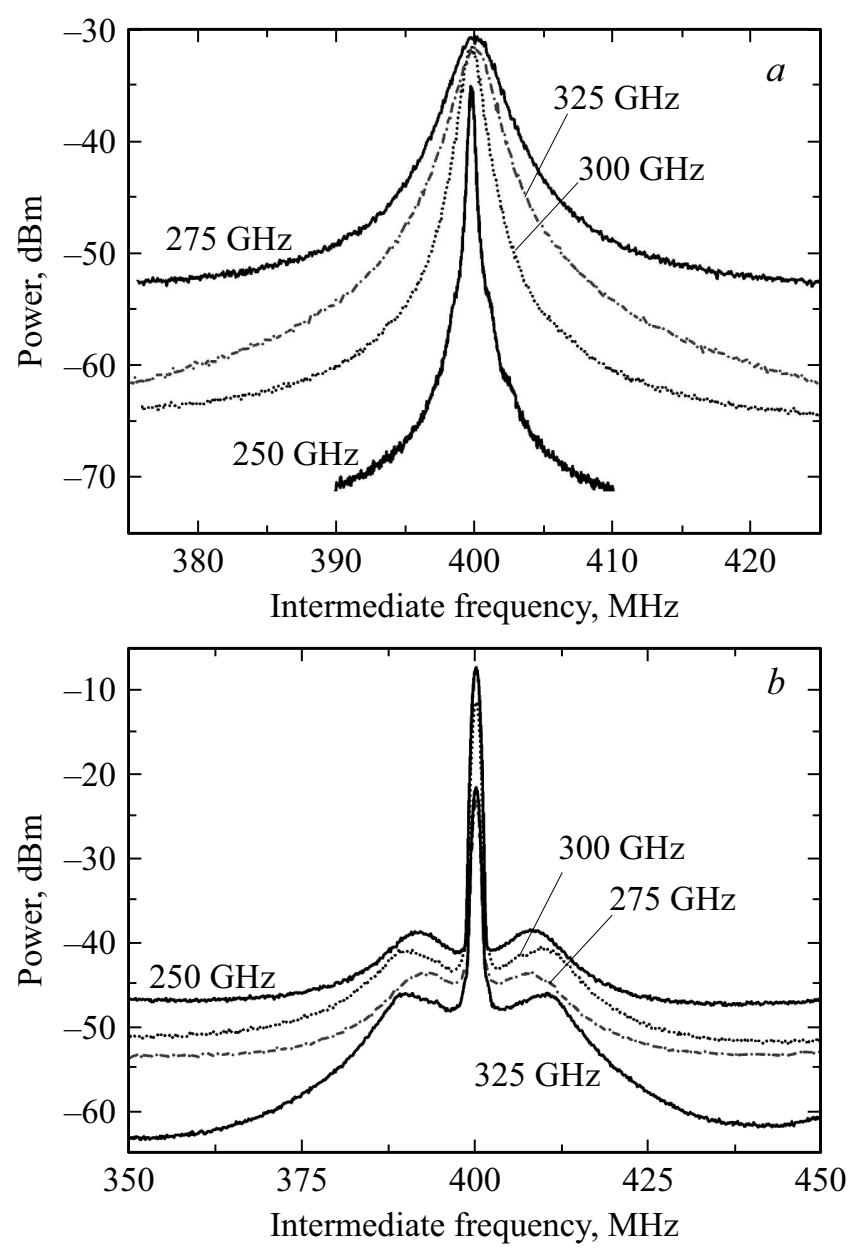

Рис. 3. Спектры излучения источника конструкции К-300, зарегистрированные при помощи анализатора спектра № 1 (см. рис. 2): $a$ ) в режиме автономной генерации; $b$ ) в режиме фазовой стабилизации при помощи системы ФАПЧ. Частота излучения $f$ для каждой АЧХ обозначены на графиках. Ширина линии генерации из $a: 0.4 \mathrm{MHz}$ для $f=250 \mathrm{GHz}, 2.9 \mathrm{MHz}$ для $f=275 \mathrm{GHz}, \quad 1.2 \mathrm{MHz}$ для $f=300 \mathrm{GHz}$, и $2.2 \mathrm{MHz}$ для $f=325 \mathrm{GHz}$. Спектральное качество из $b: 97 \%$ для $f=250 \mathrm{GHz}, 77 \%$ для $f=275 \mathrm{GHz}, 96 \%$ для $f=300 \mathrm{GHz}$, и $91 \%$ для $f=325 \mathrm{GHz}$. 


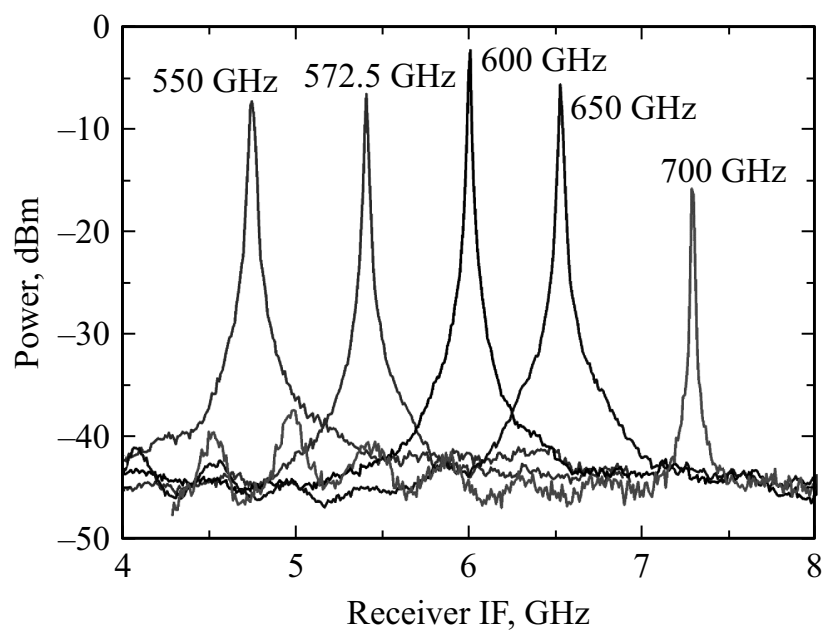

Рис. 4. Спектры излучения источника конструкции К-550, зарегистрированные при помощи анализатора спектра № 2 (см. рис. 2) в режиме автономной генерации. Частота излучения для каждой спектральной линии обозначена в подписи к кривым. Ширина линии генерации: $14.7 \mathrm{MHz}$ для $f=550 \mathrm{GHz}$, $13.7 \mathrm{MHz}$ для $f=572.5 \mathrm{GHz}, 9.4 \mathrm{MHz}$ для $f=600 \mathrm{GHz}$, $12 \mathrm{MHz}$ для $f=650 \mathrm{GHz}$, и $4.3 \mathrm{MHz}$ для $f=700 \mathrm{GHz}$.

фазовой стабилизации, определяемое как доля мощности, сосредоточенной в узком центральном пике, составила от $77 \%$ до $97 \%$. В работах [9,11] было показано, что форма спектральной линии излучения в открытое пространство соответствует форме линии излучения, измеряемой в петле обратной связи через выходной тракт гармонического смесителя, с учетом шума опорного синтезатора, который дает вклад в шум сигнала генератора, пропорциональный квадрату номера гармоники $\sim n^{2}$. Таким образом, сигнал, излученный в открытое пространство, имеет спектральные характеристики, близкие к представленным на рис. 3 для каждой частоты.

Регистрация линий излучения в открытое пространство экспериментальных образцов конструкций К-450 и К-550 проведено анализатором спектра № 2 на частотах, находящихся в рабочем диапазоне спектрометра на основе СИП. Результаты исследования приведены на рис. 4 в диапазоне ПЧ 4-8 MHz, характерная ширина линии излучения на представленных частотах составляет от $\sim 5$ до $15 \mathrm{MHz}$ в режиме автономной генерации. В работе [12] было показано, что при таких измерениях невозможно судить о мощности излучения по мощности пика регистрируемой АЧХ, поскольку эффективность преобразования СИС-смесителя в составе приемника и, следовательно, чувствительность приемника разная при каждой рабочей частоте. Поэтому мы провели исследование интегральной некалиброванной мощности излучения в открытое пространство посредством широкополосного высокочувствительного болометра на основе кремния производства Infrared Laboratories, результаты представлены в разделе 4.

\section{4. Исследование рабочего диапазона выходных частот}

Схема экспериментальной установки по исследованию рабочего диапазона выходных частот методом регистрации интегральной мощности излучения широкополосным болометром представлена на рис. 5. Как и в случае исследования АЧХ, использованы два заливных криостата с рабочей температурой $4.2 \mathrm{~K}$ : криостат № 1 с образцом исследуемого источника (верхняя часть схемы) и криостат № 2 с охлаждаемым болометром на основе кремния. Для подавления фонового инфракрасного (ИК) сигнала, к которому болометр является чувствительным, использовался кварцевый ИК-фильтр (на схеме, IR filter) на входном окне криостата № 2. Отклик болометра измерялся синхронным усилителем (на схеме, Lock-in amplifier) с использованием оптического прерывателя (на схеме, Chopper) с частотой модуляции около $170 \mathrm{~Hz}$. Исследование проводилось во всей частотной полосе перестройки РДП, при этом частота генерации определялась однозначно из соотношения Джозефсона. Точность определения частоты составляет порядка $1 \mathrm{GHz}$. Как и в случае со спектральными исследованиями, данная

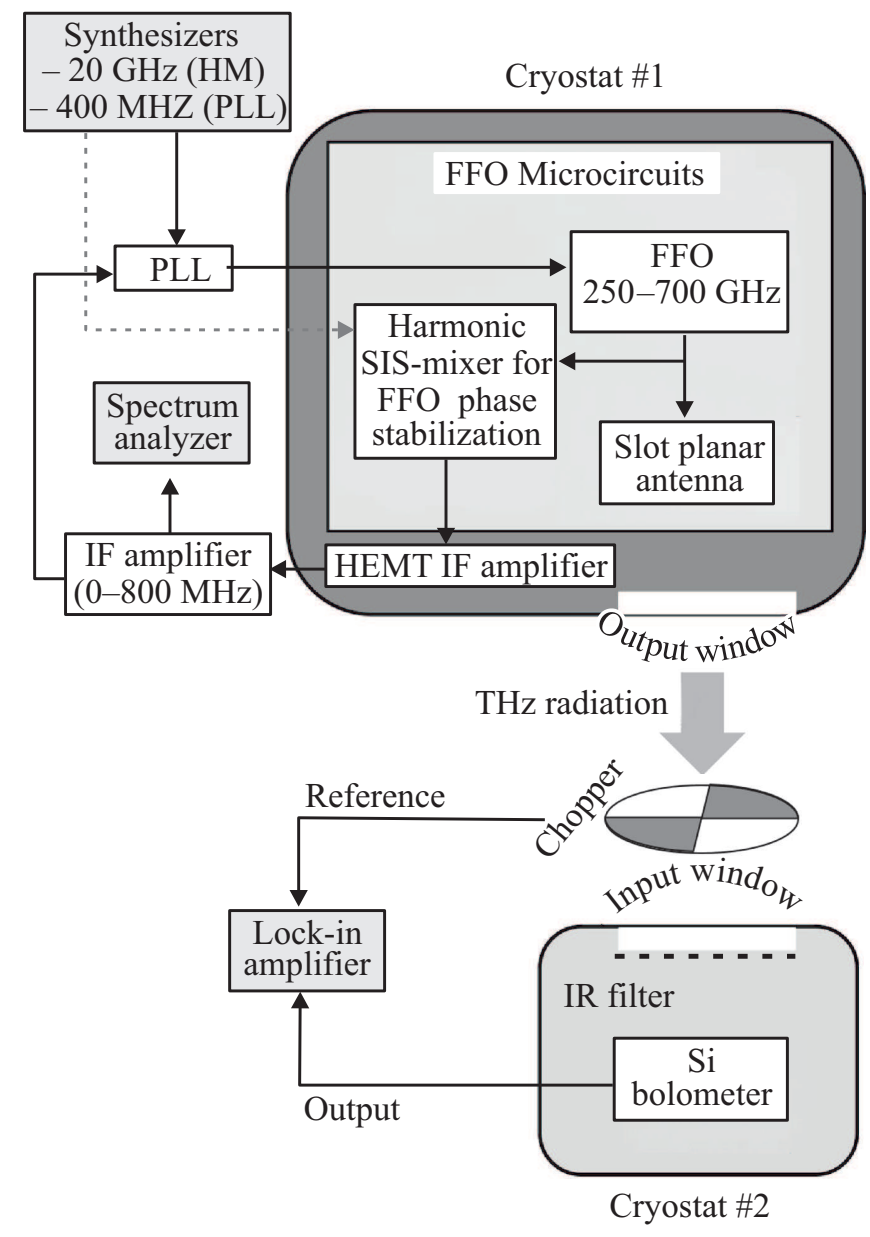

Рис. 5. Схема экспериментальной установки для регистрации выходного ТГц-излучения РДП при помощи кремниевого болометра. 


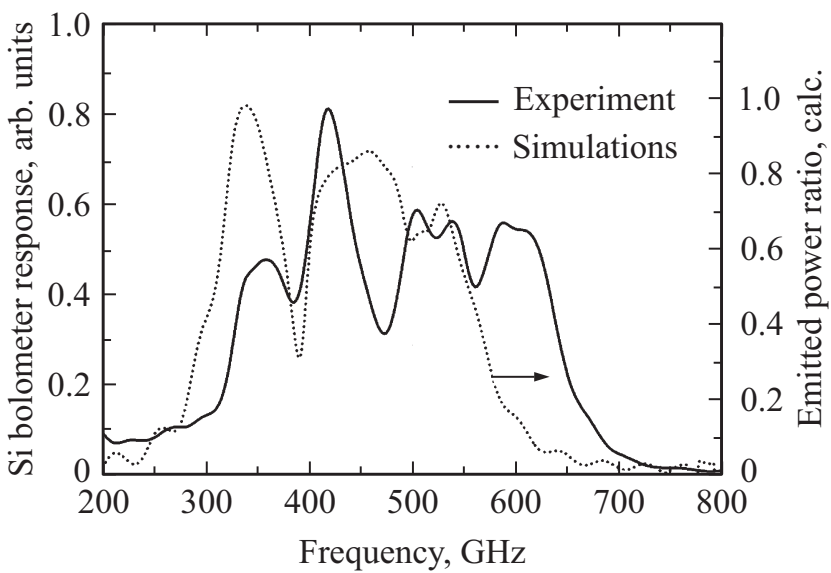

Рис. 6. Излучение источника конструкции К-550 в открытое пространство: эксперимент (сплошная кривая) и расчет (пунктирная кривая). Мощность излучения в расчете нормирована на полную выходную мощность РДП.

экспериментальная установка одновременно с измерением болометрического отклика позволяет стабилизировать излучение генератора и измерять спектральные характеристики излучения с разрешением порядка $1 \mathrm{~Hz}$, определяемым характеристиками анализатора спектра и опорного сигнала от внешнего источника СВЧ на частоте $400 \mathrm{MHz}$. Для этого предусмотрена цепь обратной связи, включающая ГС, каскад усилителей выходного сигнала ГС на промежуточной частота и систему ФАПЧ.

Экспериментальные результаты по исследованию излучения в открытое пространство представлены на рис. 6 для конструкции К-450 совместно с результатами численного моделирования. Частотная зависимость мощности излучения в открытое пространство определяется, по большей части, частотной характеристикой передающей антенны. Наблюдается качественное соответствие численных и экспериментальных результатов: наличие серии пиков и „провалов“, а также ширина рабочей полосы. Неполное соответствие обусловлено рядом упрощений, принятых при численном моделировании. Так, например, в расчетах выходная мощность РДП положена не зависящей от частоты, тогда как в реальной системе мощность зависит как от режима работы генератора $[17,18]$ (резонансный режим либо режим вязкого течения вихрей), так и от согласования излучения РДП с выходной линией передачи при конкретной частоте генерации. Кроме того, при моделировании сверхпроводниковых линий передачи бралась упрощенная модель. При численном моделировании также не учитывалось наличие гармонического состава излучения РДП. Так, например, при основной рабочей частоте $f=300 \mathrm{GHz}$, соответствующей напряжению смещения $V_{d c}=0.62 \mathrm{mV}$, имеется также генерация на второй гармонике при $f_{2 n d}=600 \mathrm{GHz}$. Рабочий диапазон перестройки частоты генерации для данной конструкции по уровню 0.5 от максимальной мощности излучения составил 340-650 GHz, при расчетных значениях 330-530 GHz. Результаты ис- следования для конструкций К-300 и К-550 также демонстрируют качественное соответствие численных и экспериментальных результатов, с учетом описанных выше факторов, не отраженных в расчетах.

\section{5. Заключение}

Источник ТГц-диапазона частот на основе РДП является перспективным типом источника для различных применений в современных терагерцовых технологиях благодаря широкому диапазону перестройки частоты для одного экспериментального образца. Нами была предложена, численно промоделирована и экспериментально исследована концепция источника излучения в открытое пространство на основе РДП, интегрированного с передающей щелевой антенной. Для фазовой стабилизации выходного излучения в схему РДП с антенной дополнительно интегрирован гармонический смеситель, обеспечивающий цепь обратной связи с генератором на основе системы ФАПЧ. Были численно промоделированы несколько конструкций интегральной микросхемы, рассчитанных на различные рабочие диапазоны частот, изготовлены и исследованы экспериментальные образцы на основе туннельных структур $\mathrm{Nb} / \mathrm{AlO}_{x} / \mathrm{Nb}$ с плотностью туннельного тока около $6.5 \mathrm{kA} / \mathrm{cm}^{2}$ и рабочей температурой $4.2 \mathrm{~K}$. Для исследования выходного излучения использованы две экспериментальные установки: ТГц-спектрометр на основе СИС-приемника со спектральным разрешением лучше $0.1 \mathrm{MHz}$ и охлаждаемый до $4.2 \mathrm{~K}$ высокочувствительный болометр на основе кремния. Ширина спектральной линии излучения в автономном режиме составила от долей $\mathrm{MHz}$ до порядка $15 \mathrm{MHz}$ в зависимости от рабочей частоты и режима работы генератора, а спектральное качество достигало 97\%. Ширина рабочей полосы источника составила более $50 \%$ от центральной частоты: например, $340-650 \mathrm{GHz}$ для конструкции К-450, что составляет $62 \%$, если положить центральной частотой $495 \mathrm{GHz}$. Это является характерным результатом для полосы пропускания двойной щелевой антенны. Общий рабочий частотный диапазон трех исследованных конструкций составил от 200 до $750 \mathrm{GHz}$.

\section{Финансирование работы}

Исследование выполнено за счет гранта Российского научного фонда (проект № 17-79-20343). Изготовление образцов выполнено с использованием уникальной научной установки (УНУ № 352529) ИРЭ им. В.А. Котельникова РАН, созданной и функционирующей за счет бюджетного финансирования в рамках государственного задания.

\section{Конфликт интересов}

Авторы заявляют, что у них нет конфликта интересов. 


\section{Список литературы}

[1] T. Nagatsuma, K. Enpuku, F. Iri, K. Yoshida. J. Appl. Phys. 54, 6, 3302 (1983).

[2] V.P. Koshelets, P.N. Dmitriev, A.B. Ermakov, A.S. Sobolev, M.Yu. Torgashin, V.V. Kurin, A.L. Pankratov, J. Mygind. IEEE Trans. Appl. Supercond. 15, 2, 964 (2005).

[3] V.P. Koshelets, S.V. Shitov, L.V. Filippenko, V.L. Vaks, J. Mygind, A.B. Baryshev, W. Luinge, N. Whyborn. Rev. Sci. Instrum. 71, 1, 289 (2000).

[4] G. de Lange, D. Boersma, J. Dercksen, P. Dmitriev, A.B. Ermakov, L.V. Filippenko, H. Golstein, R.W.M. Hoogeveen, L. de Jong, A.V. Khudchenko, N.V. Kinev, O.S. Kiselev, B. van Kuik, A. de Lange, J. van Rantwijk, A.S. Sobolev, M.Yu. Torgashin, E. de Vries, P.A. Yagoubov, V.P. Koshelets. Supercond. Sci. Technol. 23, 4, 045016 (2010).

[5] V.P. Koshelets, P.N. Dmitriev, M.I. Faley, L.V. Filippenko, K.V. Kalashnikov, N.V. Kinev, O.S. Kiselev, A.A. Artanov, K.I. Rudakov, A. de Lange, G. de Lange, V.L. Vaks, M.Y. Li, H. Wang. IEEE Trans. THz Sci. Technol. 5, 4, 687 (2015).

[6] Н.В. Кинев, К.И. Рудаков, А.М. Барышев, В.П. Кошелец. ФТT 60, 11, 2132 (2018).

[7] N.V. Kinev, K.I. Rudakov, A.M. Baryshev, V.P. Koshelets. J. Phys.: Conf. Ser. 1124, 071001 (2018).

[8] N.V. Kinev, K.I. Rudakov, L.V. Filippenko, A.M. Baryshev, V.P. Koshelets. EPJ Web Conf. 195, 02003 (2018).

[9] N.V. Kinev, K.I. Rudakov, L.V. Filippenko, A.M. Baryshev, V.P. Koshelets. J. Appl. Phys. 125, 15, 151603 (2019).

[10] N.V. Kinev, K.I. Rudakov, L.V. Filippenko, A.M. Baryshev, V.P. Koshelets. IEEE Xplore Dig. Lib.: Proc. Radiation and Scattering of Electromagnetic Waves 1, 192 (2019).

[11] Н.В. Кинев, К.И. Рудаков, Л.В. Филиппенко, А.М. Барышев, В.П. Кошелец. Радиотехника и электроника 64, 10, 970 (2019).

[12] N.V. Kinev, K.I. Rudakov, L.V. Filippenko, A.M. Baryshev, V.P. Koshelets. IEEE Trans. THz Sci. Technol. 9, 6, 557 (2019).

[13] L.V. Filippenko, S.V. Shitov, P.N. Dmitriev, A.B. Ermakov, V.P. Koshelets, J.R. Gao. IEEE Trans. Appl. Supercond. 11, 1,816 (2001).

[14] P.N. Dmitriev, I.L. Lapitskaya, L.V. Filippenko, A.B. Ermakov, S.V. Shitov, G.V. Prokopenko, S.A. Kovtonyuk, V.P. Koshelets. IEEE Trans. on Appl. Supercond. 13, 2, 107 (2003).

[15] O. Kiselev, M. Birk, A. Ermakov, L. Filippenko, H. Golstein, R.W.M. Hoogeveen, N. Kinev, B. van Kuik, A. de Lange, G. de Lange, P. Yagoubov, V. Koshelets. IEEE Trans. Appl. Supercond. 21, 3, 612 (2011).

[16] V.P. Koshelets, M. Birk, D. Boersma, J. Dercksen, P. Dmitriev, A.B. Ermakov, L.V. Filippenko, H. Golstein, R.W.M. Hoogeveen, L. de Jong, A.V. Khudchenko, N.V. Kinev, O.S. Kiselev, P.V. Kudryashov, B. van Kuik, A. de Lange, G. de Lange, I.L. Lapitsky, S.I. Pripolzin, J. van Rantwijk, A.M. Selig, A.S. Sobolev, M.Yu. Torgashin, V.L. Vaks, E. de Vries, G. Wagner, P.A. Yagoubov. Fundamentals of Superconducting Nanoelectronics. Springer, Berlin (2011). P. 263-296.

[17] D.R. Gulevich, P.N. Dmitriev, V.P. Koshelets, F.V. Kusmartsev. Nanosystems: Physics, Chemistry, Mathematics 4, 4, 507 (2013).

[18] D.R. Gulevich, V.P. Koshelets, F.V. Kusmartsev. Phys. Rev. B 96, 024515 (2017).

Редактор Е.В. Толстякова 